\title{
Detectable Anthropogenic Influence on Changes in Summer Precipitation in China $\mathscr{O}$
}

\author{
Chunhui Lu \\ National Climate Center, Laboratory for Climate Studies, China Meteorological Administration, Beijing, China
}

FRASER C. LOTT

Met Office Hadley Centre, Met Office, Exeter, United Kingdom

YING SUN

National Climate Center, Laboratory for Climate Studies, China Meteorological Administration, Beijing, and Collaborative Innovation Center on Forecast and Evaluation of Meteorological Disasters, Nanjing University of Information Science and Technology, Nanjing, China

Peter A. Stott AND Nikolaos Christidis

Met Office Hadley Centre, Met Office, Exeter, United Kingdom

(Manuscript received 17 April 2019, in final form 28 March 2020)

\begin{abstract}
In China, summer precipitation contributes a major part of the total precipitation amount in a year and has major impacts on society and human life. Whether any changes in summer precipitation are affected by external forcing on the climate system is an important issue. In this study, an optimal fingerprinting method was used to compare the observed changes of total, heavy, moderate, and light precipitation in summer derived from newly homogenized observation data with the simulations from multiple climate models participating in phase 5 of the Coupled Model Intercomparison Project (CMIP5). The results demonstrate that the anthropogenic forcing signal can be detected and separated from the natural forcing signal in the observed increase of seasonal accumulated precipitation amount for heavy precipitation in summer in China and eastern China (EC). The simulated changes in heavy precipitation are generally consistent with observed change in China but are underestimated in EC. When the changes in precipitation of different intensities are considered simultaneously, the human influence on simultaneous changes in moderate and light precipitation can be detected in China and EC in summer. Changes attributable to anthropogenic forcing explain most of the observed regional changes for all categories of summer precipitation, and natural forcing contributes little. In the future, with increasing anthropogenic influence, the attribution-constrained projection suggests that heavy precipitation in summer will increase more than that from the model raw outputs. Society may therefore face a higher risk of heavy precipitation in the future.
\end{abstract}

\section{Introduction}

Over the past few decades, notable changes have been observed in mean precipitation (Allen and Ingram 2002; Trenberth et al. 2003, 2007) and some high-impact extreme precipitation events (Westra et al. 2013; Donat

Supplemental information related to this paper is available at the Journals Online website: https://doi.org/10.1175/JCLI-D-190285.s1.

Corresponding author: Ying Sun, sunying@cma.gov.cn et al. 2016) in the context of global warming. Previous studies indicate that the atmospheric saturated water vapor pressure is related to the temperature based on the Clausius-Clapeyron equation (Trenberth et al. 2003; Hardwick Jones et al. 2010) while the changes in global mean precipitation are mainly constrained by energy balance. The changes in extreme precipitation at the global scale can be attributed to the human influence including that due to the emission of greenhouse gases (Min et al. 2008, 2009; Zhang et al. 2013). Some studies have shown that anthropogenic signal can be detected in the observed trends in extreme rainfall in the second 
half of the twentieth century (Zhang et al. 2007; Min et al. 2011; Ban et al. 2015) and the hydrologic cycle after the 1980s (Wu et al. 2013). On the basis of these studies, the Fifth Assessment Report (AR5) of the Intergovernmental Panel on Climate Change (IPCC) concluded that "anthropogenic influences have intensified extreme precipitation globally over the second half of the twentieth century with medium confidence" (Bindoff et al. 2013, p. 870).

Changes and variations in extreme precipitation have also been observed at regional scale (Qian et al. 2007; Pall et al. 2011; Christidis and Stott 2015; Sun et al. 2019). In China, many extreme rainfall events and floods have occurred in recent years during the summer, causing serious socioeconomic losses (CMA 2016, 2017). Observations also show increases in heavy precipitation and decreases in light precipitation in the second half of the twentieth century over eastern China (Zhai et al. 2005; Qian et al. 2007; Fu and Dan 2014; Ma et al. 2015). However, investigation of the anthropogenic influence on precipitation in China remains limited. The detection of precipitation at regional scales continues to be a great challenge as indicated by the fact that the current studies could not reach consensus in the human influence on precipitation in China. Some event attribution studies have found that anthropogenic-induced effects have increased the probability and risk of intense precipitation events in southeast China and north of the Yangtze River (Burke et al. 2016; Li et al. 2018; Sun et al. 2019). For the long-term changes, the anthropogenic effects on the annual changes in extreme precipitation in China were investigated using the percentile-based index (Ma et al. 2015), probability-based index (Li et al. 2017), and daily extreme rainfall index (Chen and Sun 2017). A few studies focused on questions concerning the human influence on water vapor have shown that with the enhanced warming, the increased water vapor can lead to more frequent extreme weather and climate events (Zhao et al. 2016; Zhang et al. 2019; Zhang and Zhao 2019). Burke and Stott (2017) split the annual rainfall record in China into two halves and found that human-induced climate change led to an overall decrease in monsoon rainfall and an increase in dry days in past decades. While these studies have explored various aspects of the human influence on precipitation changes in China, low signal-to-noise ratios (Zhang et al. 2013) have made it difficult to detect the anthropogenic signals in the regional and seasonal precipitation changes up to now. Here, we investigate the human influence on summer precipitation in China, from the viewpoint of changes in precipitation amount in different intensity categories, to improve our understanding of climate model performance and make reasonable projection of future seasonal precipitation changes.
We use daily precipitation data (Yang and Li 2014) in China and model outputs from phase 5 of the Climate Model Intercomparison Project (CMIP5) (Taylor et al. 2012) to classify summer precipitation into three categories based on the rainfall amount. The precipitation changes in one category could affect the changes in another category either through changes in frequency or event magnitude. Based on the Clausius-Clapeyron relation between water vapor and temperature, (Trenberth 1998; Semenov and Bengtsson 2002; Trenberth et al. 2003), there could be an increase in heavy precipitation and decrease in light precipitation with the warming, due to energy budget constraints. Thus we also conduct a joint three-category detection and attribution analysis, as well as a detection analysis of precipitation change in each category, to deal with the covariation and dependence between the three categories. Using the detection and attribution results, we produce and analyze observationally constrained future projections of Chinese summer precipitation. The remainder of this paper is organized as follows: sections 2 and 3 describe the data and detection methods, including data processing; section 4 presents the primary results; and the conclusions of this study and some discussion are provided in section 5 .

\section{Data}

\section{a. Observational data and precipitation classifications}

Daily precipitation collected at 2419 stations across China was obtained from China's National Meteorological Information Center. All of these data have been quality controlled and adjusted for homogeneity using the RHtest software package (Xu et al. 2013; Wang and Feng 2010; Yang and Li 2014). The data quality and number of stations in this dataset are stable after 1961 (Fig. S1a in the online supplemental material) so our analyses are focused on the period of 1961-2012.

Based on China's national standard for precipitation classification (CMA 2012) issued by the China Meteorological Administration, we classify precipitation into three categories based on daily rates: light precipitation $\left(1-10 \mathrm{~mm} \mathrm{day}^{-1}\right)$, moderate precipitation $\left(10-25 \mathrm{~mm}\right.$ day $\left.^{-1}\right)$, and heavy precipitation $\left(>25 \mathrm{~mm}\right.$ day $\left.^{-1}\right)$. We calculate the seasonal precipitation accumulation in each category. A precipitation ratio was computed by dividing the number of days with light, moderate, or heavy precipitation by the total number of rain days, with data expressed as a percentage. Mean precipitation intensities were calculated as the mean precipitation rate over days with light, moderate, heavy, or total precipitation. 
This classification might impede detection in some parts of China due to the skewed distribution of precipitation. Nevertheless, changes in different categories of precipitation could have important implications on different sectors of society and ecosystems. The occurrence of precipitation with different fixed thresholds could have great and severe impacts on urban drainage systems and flood control capacity, as well as agricultural practices. Also, from the viewpoint of detection and attribution, the anthropogenic influence on heavy precipitation seems to be expected. Precipitation has a skewed distribution, with a fixed lower bound at zero and a long unbounded upper tail. If anthropogenic influence acts to increase precipitation, one would expect the distribution to stretch out toward the right. Intuitively, one might expect the most-discernable changes to become evident in the upper tail (although uncertainty in estimating change in the upper tail may adversely affect detection). This thus leads us to investigate the categories in which anthropogenic influence can be detected and how reasonable the current operational classification of precipitation is from the viewpoint of long-term climate changes.

\section{b. Model simulations}

CMIP5 multimodel simulations were used to estimate the Chinese summer precipitation responses to external forcing and the internal climate variability of summer mean precipitation. The three precipitation categories were computed based on daily data from individual simulations of different climate models. Details of the model simulations used in this study are listed in Table S1 in the online supplemental material (acronym expansions and definitions are available at https:// www.ametsoc.org/PubsAcronymList). We used 68 historical simulations from 21 climate models to represent the response to combined anthropogenic and natural forcing (ALL). Because most of these simulations ended in 2005, the RCP8.5 simulated results for 2006-12 were used to extend the ALL forcing data to 2012. In addition, $49 \mathrm{RCP} 4.5$ and RCP8.5 simulations from 21 models were used to estimate future changes from 2013-2100. Additionally, 36 simulations from 11 models driven only by natural forcing (NAT) and 20 simulations from 6 models driven by greenhouse gas forcing only (GHG) were used in this research, with all the simulations ending in 2012. We estimated the anthropogenic (ANT) response as the difference between the ALL and NAT responses, assuming that the responses to anthropogenic and natural forcing combine linearly. To evaluate the internal climate variability, preindustrial control (CTL) simulations based on 31 models were used to calculate the three categories of summer precipitation, with 302 pieces of 52-yr chunks.

\section{Methods and data processing}

\section{a. Detection methods}

We compare the spatiotemporal evolution of summer precipitation in the observations and model simulations using a standard optimal fingerprinting technique (Allen and Stott 2003). This method assumes that observations $\mathbf{Y}$ can be expressed as the sum of scaled fingerprints $\mathbf{X}$ plus the internal climate variability $\boldsymbol{\varepsilon}: \mathbf{Y}=(\mathbf{X}-\mathbf{v}) \beta+\boldsymbol{\varepsilon}$. Here, $\varepsilon$ represents the residual or internal variability in the observations and $\mathbf{v}$ represents noise in the signal(s) $\mathbf{X}$. The estimated scaling factor $\beta$ adjusts the signal estimate $\mathbf{X}-\mathbf{v}$ that is produced by the total least squares algorithm and can be used to scale the response patterns as well as provide a good match with the observations. The covariance matrix $\varepsilon$ can be estimated from CTL simulations, and the scaling factor $\beta$ can be estimated based on the implementation of the optimal fingerprint method of Ribes and Terray (2013). Detection of a signal is claimed if the $90 \%$ confidence interval of the corresponding scaling factor lies entirely above zero. Moreover, scaling factors consistent with 1 suggest good agreement between the observed values and model results.

We conduct two regression analyses, a single-signal and a two-signal analysis, to assess the relative roles of individual forcing mechanisms in relation to the observed changes. In the single-signal analysis, the observations are regressed onto the multimodel mean responses of single factors. This approach allows us to assess whether there is evidence that the response of a specific forcing might be present the observed changes or whether the observations contain the response to a specified combination of forcings such as the ALL signal. In the two-signal analyses, the observed changes are partitioned between the simulated responses to natural forcing (NAT), anthropogenic forcing (ANT), and internal variability. Observations are organized into a vector $\mathbf{Y}$, and the ALL and NAT responses are also organized into vectors $\mathbf{X}_{\mathrm{ALL}}$ and $\mathbf{X}_{\mathrm{NAT}}$. Assuming that the ANT forcing response $\left(\mathbf{X}_{\mathrm{ANT}}\right)$ can be approximated by the difference between $\mathbf{X}_{\mathrm{ALL}}$ and $\mathbf{X}_{\mathrm{NAT}}$, we obtain the following expression:

$$
\begin{aligned}
\mathbf{Y} & =\left(\mathbf{X}_{\mathrm{ALL}}-\mathbf{v} 1\right) \beta_{1}+\left(\mathbf{X}_{\mathrm{NAT}}-\mathbf{v} 2\right) \beta_{2}+\varepsilon \\
& =\left(\mathbf{X}_{\mathrm{ANT}}+\mathbf{X}_{\mathrm{NAT}}-\mathbf{v} 1\right) \beta_{1}+\left(\mathbf{X}_{\mathrm{NAT}}-\mathbf{v} 2\right) \beta_{2}+\varepsilon,
\end{aligned}
$$

where the noise term $\varepsilon$ represents the effects of internal variability. This analysis aims to estimate the scaling factors associated with the ANT and NAT responses: $\beta_{\mathrm{ANT}}=\beta_{1}$ and $\beta_{\mathrm{NAT}}=\beta_{1}+\beta_{2}$. Thus, if the model provides satisfactory detection results, it may be possible to estimate the separate contributions of these two 
factors to climate change. All available model simulations for the ALL and NAT forcings are used to compute the response to the ANT forcing. Differences in the collections of models used to estimate the ALL and NAT signals may affect the estimate of the response to ANT forcing. Therefore, the same detection analyses are repeated using the eight models that provide both ALL and NAT simulations. Similar detection results (Fig. S2 in the online supplemental material) are obtained when using all available models, irrespective of whether they provide NAT simulations, suggesting little influence of the different sets of models. Because detection and attribution assessments largely depend on model simulations, it is important to evaluate the model simulation results compared to observations to increase confidence in the robustness of the attribution results. We perform a power spectra analysis for the modeled and observed changes to determine whether the models adequately estimate observed variability. Simultaneously, a residual consistency test is performed according to the implementation given by Ribes and Terray (2013).

\section{b. Data processing}

Different categories of precipitation were separately classified for individual stations. The seasonal accumulated precipitation amount was calculated for each category based on daily data and then the station anomalies were calculated by removing the 1961-90 means. These anomalous values were aggregated to produce gridded data with a $3^{\circ} \times 3^{\circ}$ resolution by averaging all available observations within each grid cell. Each grid cell has a minimum of three observing stations (Figs. S1b-d in the online supplemental material). The gridded results were subsequently used to calculate the area-weighted regional mean anomalies. Simultaneously, all the model data were classified into three precipitation categories to obtain the accumulated precipitation amounts of different categories in summer. Anomalies were calculated relative to the 1961-90 means and then interpolated to the same $3^{\circ} \times 3^{\circ}$ grid as the observations.

To make the noise covariance matrix full rank, as needed for optimal detection analyses, 5-yr nonoverlapping means were used to reduce the temporal dimension. Regional means, rather than grid cell series, were used to further reduce the spatial dimension. Due to different regional climate feature across China, with eastern China (EC; east of $105^{\circ} \mathrm{E}$ ) having a typical monsoon climate and western China (WC; west of $105^{\circ} \mathrm{E}$ ) an arid or semiarid climate, we conducted the detection analyses for all of China as a single region and also divide it into EC and WC subregions. The model data were masked based on the availability of the observations prior to the calculation of the regional means. Ensemble means were first computed for individual model runs and then averaged over all available models. Linear trends in the time series of summer precipitation from 1961-2012 were evaluated based on the regional averages using the classical least squares regression method. The number of dimensions was 11 for each regional series, made up of 10 nonoverlapping 5-yr means and one additional 2-yr mean. This mixture may lead the last point having a larger variance, which is accounted for in the estimate of the variance-covariance matrix of internal variability. Detection analyses were conducted on the three categories of precipitation, and the data of heavy, moderate, and light precipitation were then combined into a joint three-category precipitation series to investigate the human influence on them simultaneously.

All available data from the within-ensemble difference of simulations and CTL simulations were used to estimate the internal variability. The within-ensemble difference and control run data were split into two equal independent sets with 213 fifty-two-year chunks of noise data in each set. We use these two sets for optimization to obtain the best estimate of the scaling factor $\beta$, estimate the uncertainty range of $\beta$, and conduct the residual consistency test. The large sample of simulated internal variability in this research provides increase confidence in the estimates of the covariance structure of internal variability.

\section{Results}

\section{a. Spatial distribution of changes in summer precipitation}

Figure 1 (left column) shows observed linear trends of summer (June-August) accumulated precipitation amounts for total, heavy, moderate, and light wet day precipitation. Increasing trends are observed for total and heavy precipitation in southeastern China and northwestern China. There is a drying belt from southwestern China to northeastern China. For moderate precipitation, regional differences are obvious, especially significant negative trends are observed in northeast and southwest regions. For light precipitation, most parts of the country have experienced decreasing trends, especially in EC. The increase of total precipitation in southeastern China is mainly related to the increase of heavy precipitation in the region while the increase of total precipitation in northwestern China is mainly due to increases in light and moderate precipitation.

The model simulated ensemble means of linear trends in seasonal accumulated precipitation amounts calculated from individual runs under ALL forcing are exhibited in the second column of Fig. 1. It is clear that total and heavy precipitation increases almost everywhere, which is 

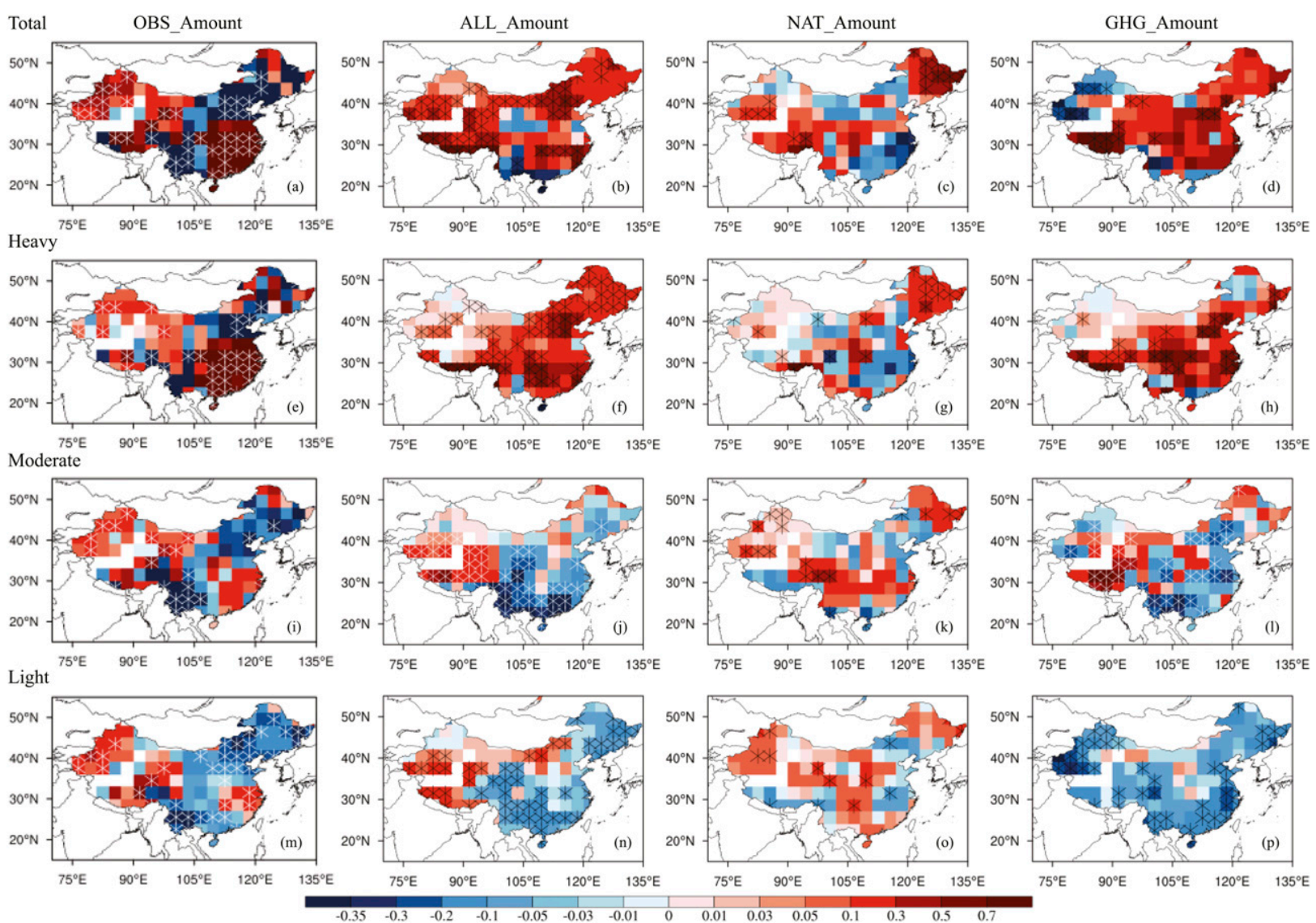

FIG. 1. Trends in the summer precipitation amount anomalies $\left(\mathrm{mm} \mathrm{yr}^{-1}\right)$ for the (a)-(d) total, (e)-(h) heavy, (i)-(l) moderate, and (m)-(p) light precipitation from 1961 to 2012 as based on (left) observations and the model-simulated responses to the (left center) ALL forcing, (right center) NAT forcing, and (right) GHG forcing. A grid cell is marked with an asterisk if the trends are statistically significant at the $10 \%$ level according to the Student's $t$ test. White grid cells denote that there are no observation in that area.

roughly consistent with the observations. The models could not reproduce the decreasing trends of total and heavy precipitation from southwestern China to northeastern China. The responses of total and heavy precipitation to ALL forcing in northeastern China are opposite to the observations. For moderate precipitation, the models reproduce the increase of moderate precipitation in northwestern China and decrease in northeastern China but fail to reproduce the increase of precipitation in central part of China. The simulation of light precipitation in models is better than for other categories, with models reproducing well the observed decrease of light precipitation in eastern China and increase of light precipitation in western China. The model responses to NAT forcing show some different features. The NAT results exhibit opposite trends to observations for total, heavy, and light precipitation in EC. Especially for the total precipitation, large magnitude changes but with opposite sign to that of observations can be found in eastern and southwestern China, suggesting that the modeled response to NAT forcing is opposite to the observed change in total precipitation. Responses of precipitation in all categories to GHG forcing (right column) are quite similar to those under ALL forcing, indicating an important role of GHG forcing in the changes of summer precipitation in China.

To further understand the characteristics of precipitation amount, Fig. 2 shows the histograms of daily precipitation frequency and amount derived from observations and model simulations under ALL forcing for the period of 1961-2012. The regional mean histograms of China, EC, and WC are first estimated at each grid box and then averaged in the region. Figure 2a shows that the models generally reproduce the distribution of light and moderate precipitation in China, but slightly underestimate frequency for precipitation events more intense than $60 \mathrm{~mm} \mathrm{day}^{-1}$. This is also shown in the distribution of precipitation amount in each bin (Fig. 2b). In contrast, the models underestimate the frequency and amount for precipitation events more intense than $20 \mathrm{~mm} \mathrm{day}^{-1}$ in 

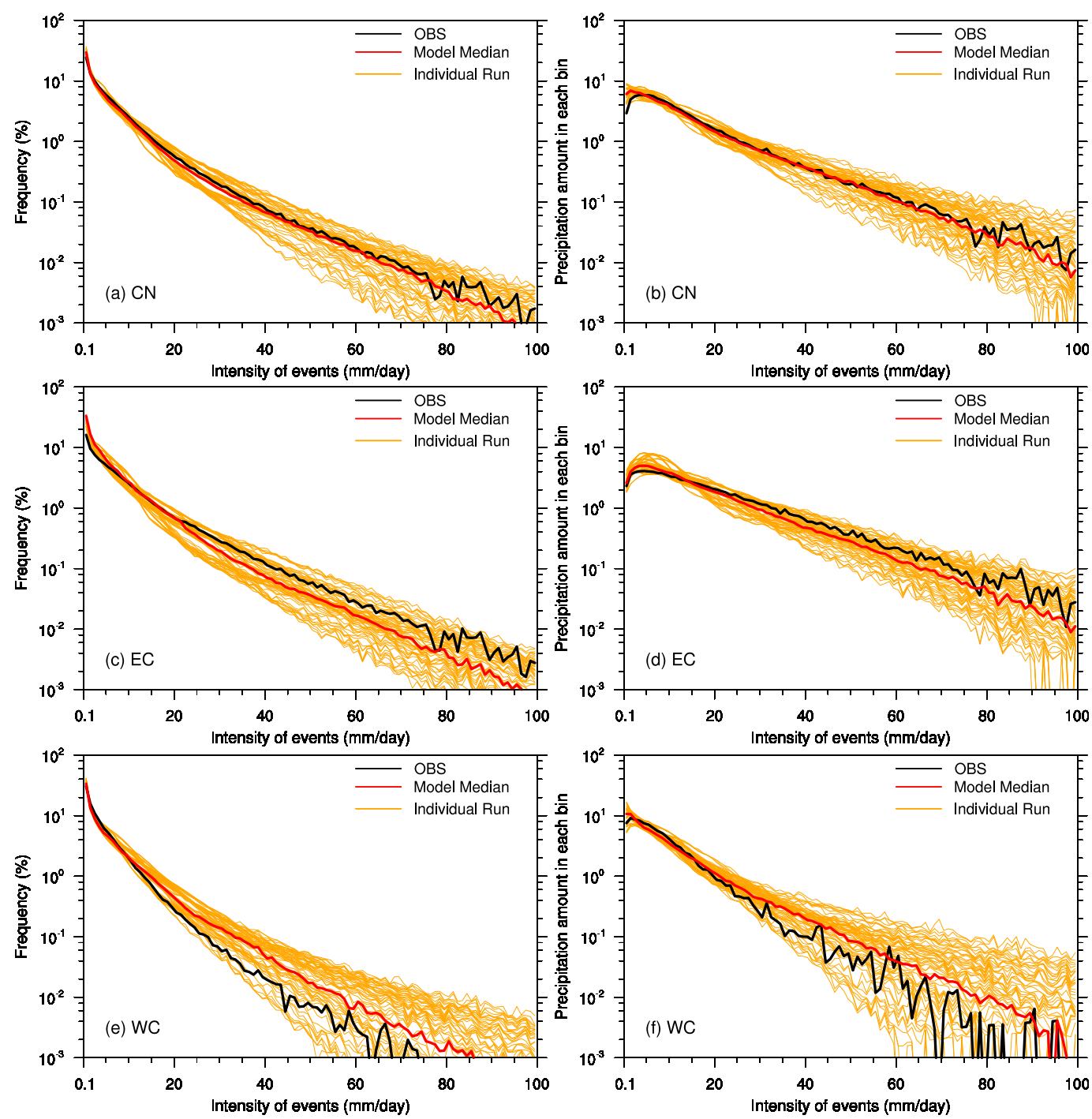

FIG. 2. Distribution of daily precipitation (left) frequency and (right) amount ( $\mathrm{mm}$ ) in each bin as a function of precipitation intensity (bin size is $1 \mathrm{~mm} \mathrm{day}^{-1}$ ) from observations (black traces; 1961-2012) and model simulations (red traces indicate the multimodel median, and orange traces indicate results from individual runs) averaged over (a),(b) China, (c),(d) EC, and (e),(f) WC.

EC (Figs. 2c,d) and overestimate them in WC (Figs. 2e,f). This suggests that the models roughly reproduce the distributions of daily precipitation frequency and amount in China but some biases exist in EC and WC. Especially in WC, the models show poor performance in simulating most of the precipitation distribution. We will mainly focus on the detection analyses in China and EC hereafter.

\section{b. Regional averaged time series of summer precipitation}

Figures 3 and 4 show the observed and model simulated temporal evolution of summer precipitation from four categories of precipitation. The trends in the regional mean precipitation for the period 1961-2012 are shown in the right column. The observed total and heavy precipitation are characterized by little change in the early period and an apparent increase after the late 1980s in China and EC. The changes in total and heavy precipitation are larger than those in moderate and light precipitation. These characteristics are successfully reproduced by the model simulations under ALL forcing. The observed series mostly fall within the range of ALL simulations, suggesting models perform well in reproducing these changes. The response to GHG forcing exhibits similar temporal variations, but has larger trends than those under ALL forcing for all categories of precipitation. The trends in the NAT simulations are not consistent with observations for light and moderate precipitation (Figs. 3f,h and 4f,h). 

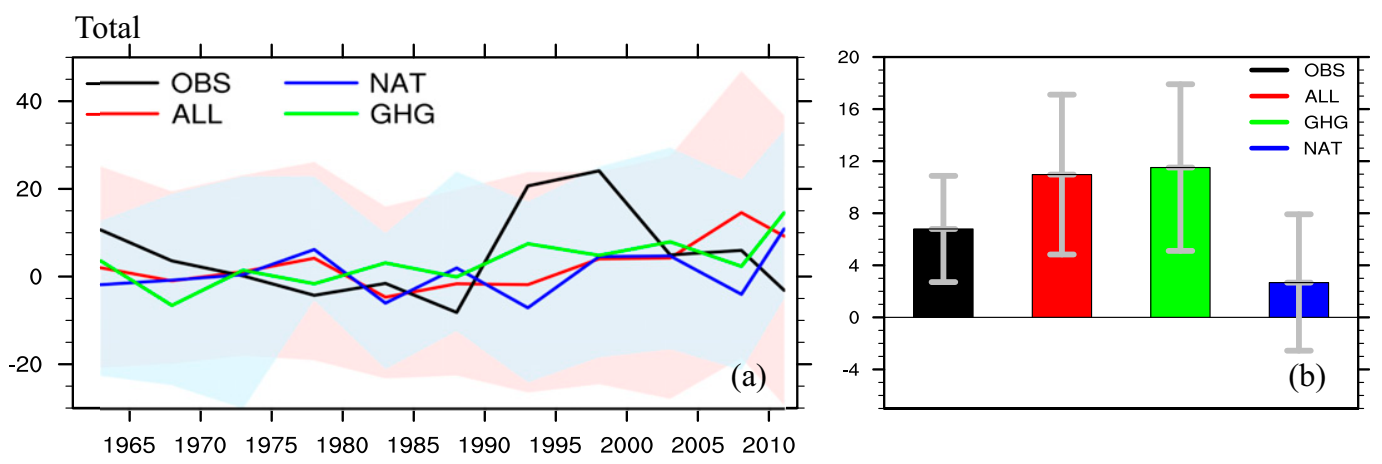

Heavy
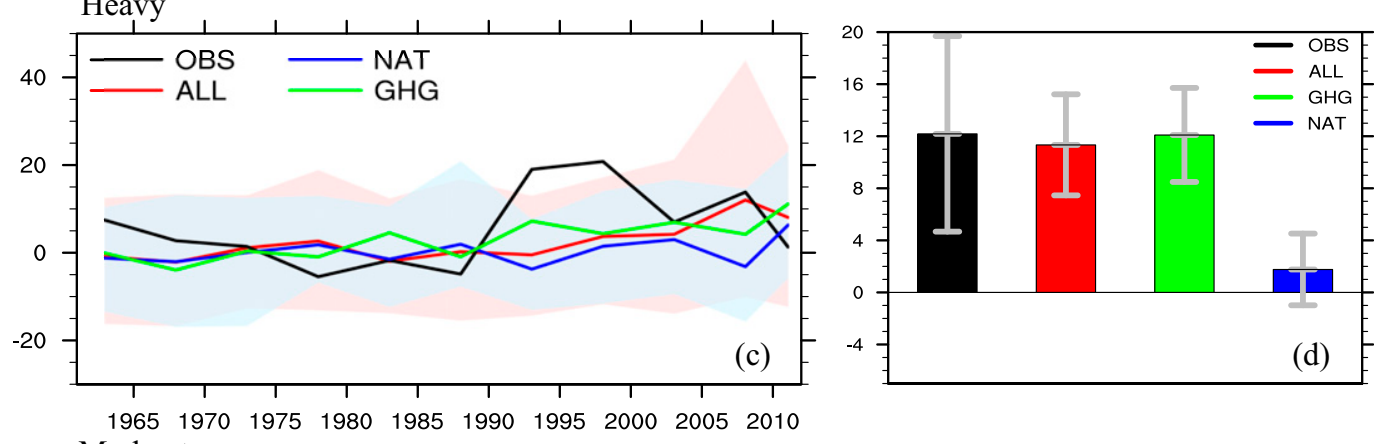

Moderate
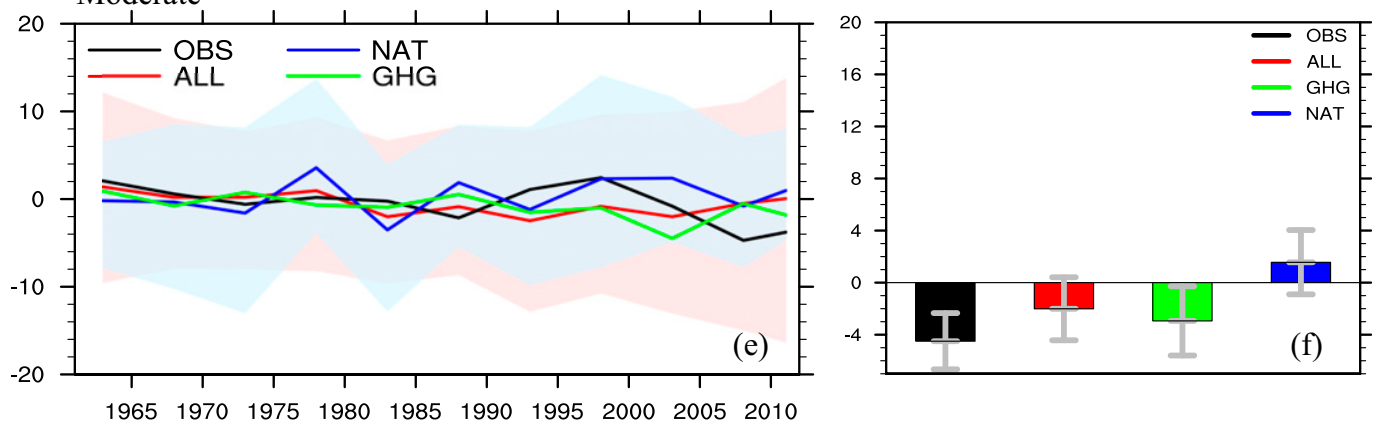

Light
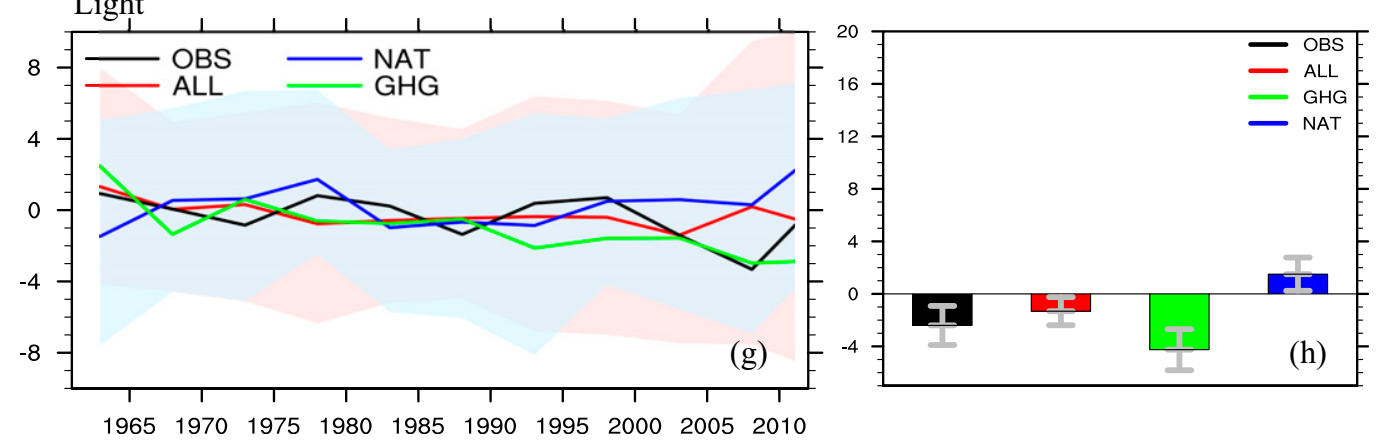

FIG. 3. (left) Time series of 5-yr mean anomaly values (relative to 1961-90) averaged over all of China for the (a) total, (c) heavy, (e) moderate, and (g) light precipitation. The solid lines show the observation or ensemble means of the model simulations, and the shaded areas show the $5 \%-95 \%$ ranges of model simulations. Red, green, and blue denote model simulations under ALL, GHG, and NAT forcings, respectively. (right) Their respective long-term trends (color bars) and the corresponding $90 \%$ confidence intervals (gray error bars). 

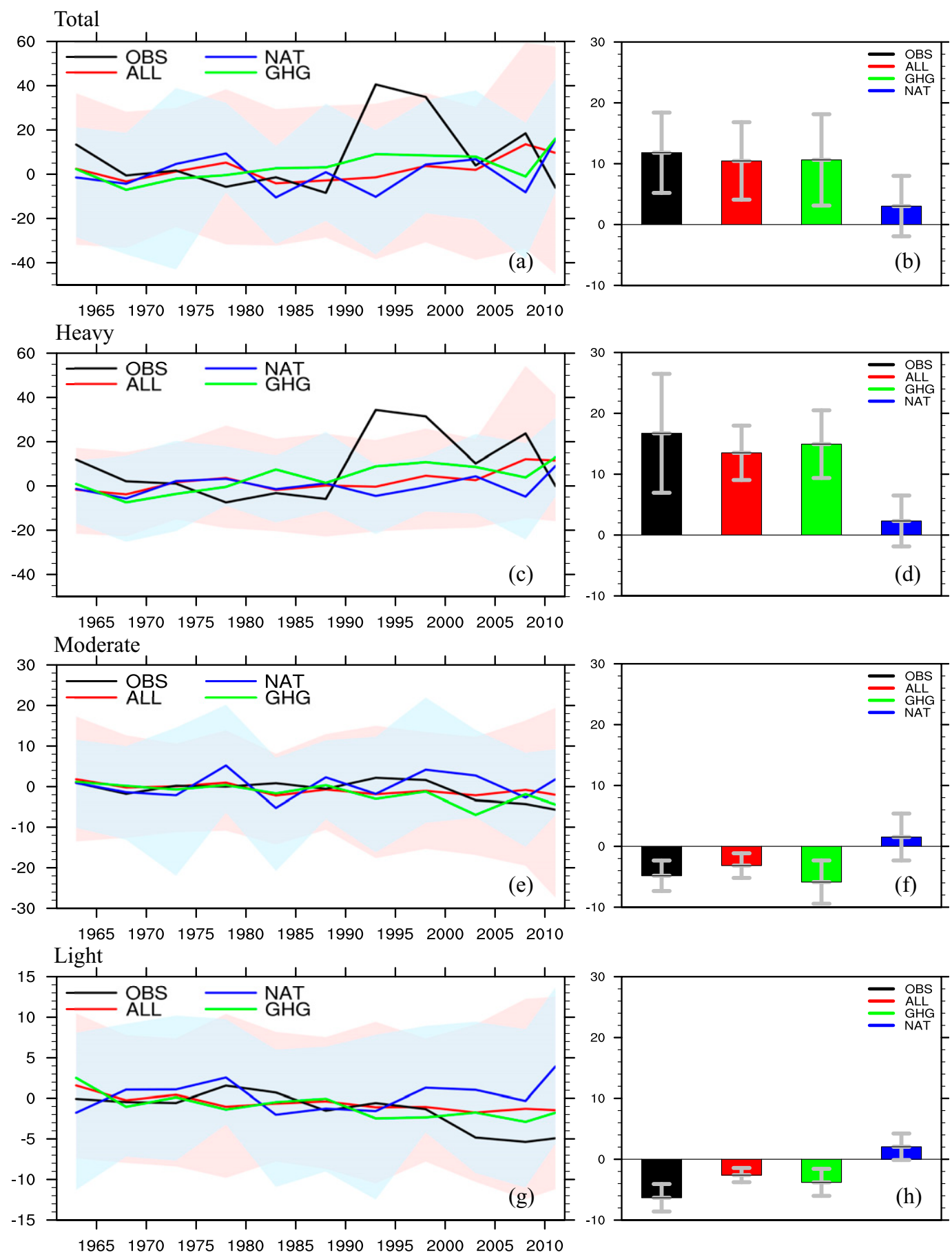

FIG. 4. As in Fig. 3, but for the regional mean averaged over eastern China.

As shown in Fig. 1, the observed precipitation trend has a similar magnitude to the modeled trend based on the ALL forcing, but the modeled trend based on the NAT forcing is much weaker for total and heavy precipitation and of the opposite sign for moderate and light precipitation. The GHG results have the same sign as those of ALL, but with a larger magnitude of changes. On the subnational scale, the precipitation category trends in EC have high magnitudes, especially that for heavy precipitation, which increased by approximately $17 \mathrm{~mm}$ over the 52-yr period. The modeled trend under the ALL forcing has the same sign as for the observations 

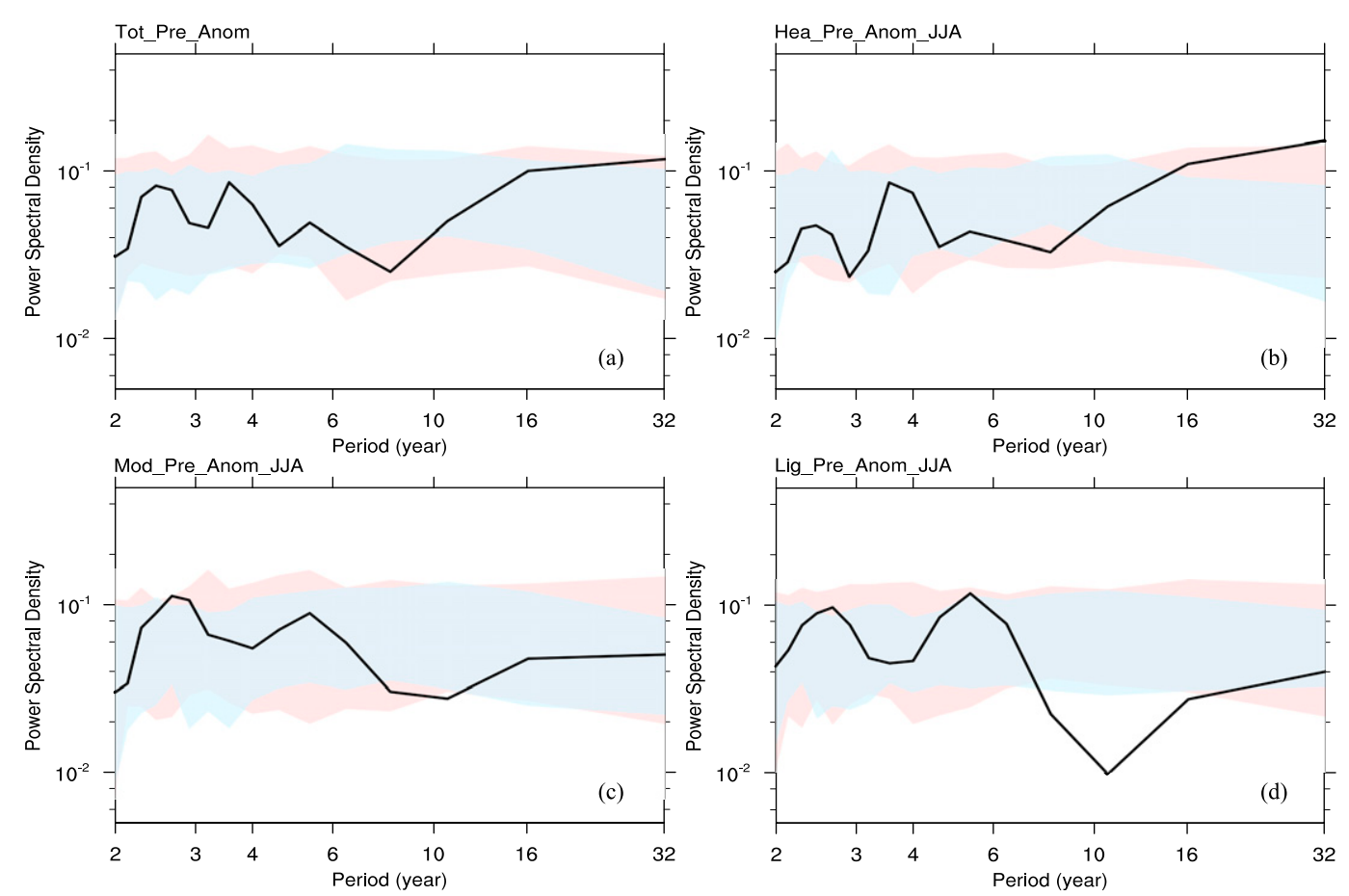

FIG. 5. Power spectral density of the (a) total, (b) heavy, (c) moderate, and (d) light precipitation anomalies in China from 1961 to 2012 for the observations (black) and model simulations. All model data have the same spatial coverage as the observations. The 5\%-95\% ranges of the historical ALL (pink) and historical NAT (blue) multimodel ensemble are shown as shaded areas.

but has a smaller magnitude. The characteristics of the modeled trend under the NAT and GHG forcings are similar to those at the national level in China.

The observed and multimodel simulated power spectra of the four categories of precipitation are shown in Fig. 5 (see Figs. S3-S6 in the online supplemental material for the power spectra from simulations by individual models). We computed the power spectra based on the time series for China from 1961-2012 from observations and separately from each simulation in the ALL and NAT experiments. The power spectra of the observations for total, heavy and moderate precipitation are found to lie within the range of the power spectra from the ALL simulations at all the time scales examined, suggesting that the simulated variability is reasonably consistent with that of the observations. This supports the use of these model simulations for detection and attribution analyses. There is some indication that model simulated variability is greater than observed for the light precipitation category at the interdecadal time scale, which should not materially affect the detection and attribution analyses in this study.

\section{c. Detection results}

Figure 6 displays the best estimates and their 90\% confidence intervals for the scaling factors from the single-signal (ALL and GHG) and two-signal (ANT and NAT) analyses in China and EC. For China as a whole (CN), the ALL signal is robustly detected in the heavy and the joint three-category precipitation series, and the residual consistency test is passed. The scaling factors for the ALL signal are significantly greater than zero (at the $5 \%$ significance level), and the $90 \%$ confidence intervals include 1 , indicating that the observed changes are generally consistent with the simulated responses to the combined influences of anthropogenic and natural forcings simulated by the models. For the moderate and light precipitation categories, the ALL signal cannot be detected, as the $90 \%$ confidence intervals include zero. In EC, the ALL signal can also be robustly detected in the heavy and joint three-category precipitation series but with large uncertainty ranges for the scaling factors. Additionally, the model-simulated responses to ALL forcing underestimate the observed changes in both heavy and combined precipitation, with the scaling factor lying entirely or mostly above 1 . Note that the GHG signal can be detected in one-signal analyses of the total and heavy precipitation series in $\mathrm{CN}$ and $\mathrm{EC}$, as the $90 \%$ confidence intervals lie above zero, indicating that the GHG forcing is of great importance in the changes of summer precipitation during 1961-2012 in China. 

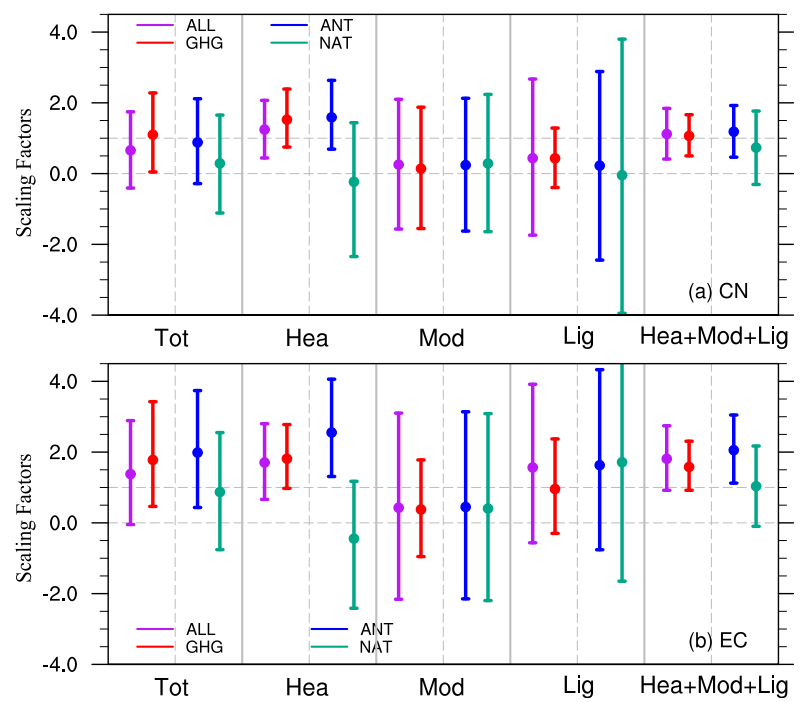

FIG. 6. Best estimates of the scaling factors and their 5\%-95\% uncertainty ranges for the 5-yr mean total, heavy, moderate, light, and combined precipitation in summer as based on single-signal (ALL and GHG) and two-signal (ANT and NAT) analyses in (a) China and (b) eastern China.

For the two-signal detection, the $90 \%$ confidence intervals of the ANT scaling factors for heavy and joint three-category precipitation lie above 0 in China and above 1 in EC. This indicates that the ANT signal can be detected in heavy and joint three-category precipitation in these two domains when the influence of NAT is taken into account. Heavy precipitation is underestimated in EC, which is consistent with the single-signal results. For the moderate and light precipitation, the ANT and NAT signals could not be detected.

The signal-to-noise ratios are also estimated. The linear trends in precipitation changes are considered as signals and the signal-to-noise ratios are then estimated by dividing the linear trends by the standard deviation during 1961-2012, based on the residual series after removing the trends in the models (Table 1; also see the online supplemental material). For the total, moderate, and light precipitation, low signal-to-noise ratios are found in three regions mainly due to large variability and small signal. In contrast, for the heavy and joint three-category precipitation, the signal-to-noise ratios are larger when compared with the other categories. This partially explains why the human influence on heavy and joint three-category precipitation can be detected.

We further quantify the attributable trends from the ALL and ANT signals to the observed 52-yr changes in heavy precipitation based on the corresponding scaling factors calculated from the combined series. The heavy precipitation averaged in China increased by approximately $13 \mathrm{~mm}$ from 1961 to 2012 . The best estimates of the increase attributable to ALL and ANT forcings are respectively approximately $12 \mathrm{~mm}$ (with a $90 \%$ confidence interval of 5-19 mm) and $11 \mathrm{~mm}$ (with a $90 \%$ confidence interval of $6-16 \mathrm{~mm}$ ). Similar results can be found in the EC with high-magnitude variational trends.

\section{d. Observation-constrained future projections}

The best estimate and its $90 \%$ confidence intervals of the scaling factor for the ALL signal in China are used to scale the multimodel projections under the RCP4.5 and RCP8.5 scenarios from 2013 to 2100 . The observationally constrained technique based on detection results, which is generally called the "ASK" technique, has been used in previous studies (including Allen et al. 2000; Stott et al. 2006; Stott and Jones 2012; Huntingford 2013; Jones et al. 2016). The main idea is that if a model over- or underestimates the response of climate system to external forcing during the historical period, it is assumed that it will continue to similarly over or underestimate the climate response in the future. Thus the detection results can be used to constrain the future projection. Based on our single- and two-signal detection analyses, the CMIP5 model-simulated underestimate the observed changes in heavy precipitation in China and EC. The scaled future projections in heavy precipitation (dashed lines in Fig. 7) project heavier precipitation than the models themselves, especially in EC. Under the RCP8.5 scenario, the best estimate of the precipitation increase in EC in 2040 is approximately $29 \mathrm{~mm}$ (90\% confidence interval from 17 to $41 \mathrm{~mm}$ according to the confidence intervals of the scaling factor), which is greater than the direct multimodel mean projection of $24 \mathrm{~mm}$. The RCP 4.5 results are quite similar. Given the underestimation of the increasing trend in heavy precipitation by the CMIP5 models, society may therefore face a higher possibility of occurrence

TABLE 1. Signal-to-noise ( $/ \mathrm{N})$ ratios of the total, heavy, moderate, light, and combined precipitation anomalies (the calculation method is shown in detail in the online supplemental material).

\begin{tabular}{|c|c|c|c|c|c|c|c|c|c|c|c|c|c|c|c|}
\hline & \multicolumn{3}{|c|}{ Total } & \multicolumn{3}{|c|}{ Heavy } & \multicolumn{3}{|c|}{ Moderate } & \multicolumn{3}{|c|}{ Light } & \multicolumn{3}{|c|}{ Combined } \\
\hline & $\mathrm{CN}$ & $\mathrm{EC}$ & WC & $\mathrm{CN}$ & $\mathrm{EC}$ & WC & $\mathrm{CN}$ & $\mathrm{EC}$ & WC & $\mathrm{CN}$ & $\mathrm{EC}$ & WC & $\mathrm{CN}$ & $\mathrm{EC}$ & WC \\
\hline Signal & 10.97 & 10.46 & 10.9 & 11.34 & 13.52 & 9.1 & 2.01 & 3.16 & 1.66 & 1.26 & 2.6 & 0.25 & 7.28 & 8.84 & $\overline{5.53}$ \\
\hline Noise & 10.61 & 11.37 & 10.3 & 6.38 & 8.83 & 7.81 & 4.37 & 6.49 & 4.49 & 2.99 & 4.56 & 3.30 & 3.82 & 5.88 & 5.55 \\
\hline $\mathrm{S} / \mathrm{N}$ ratio & 1.03 & 0.91 & 1.06 & 1.78 & 1.53 & 1.16 & 0.45 & 0.48 & 0.37 & 0.42 & 0.57 & 0.08 & 1.91 & 1.50 & 0.99 \\
\hline
\end{tabular}



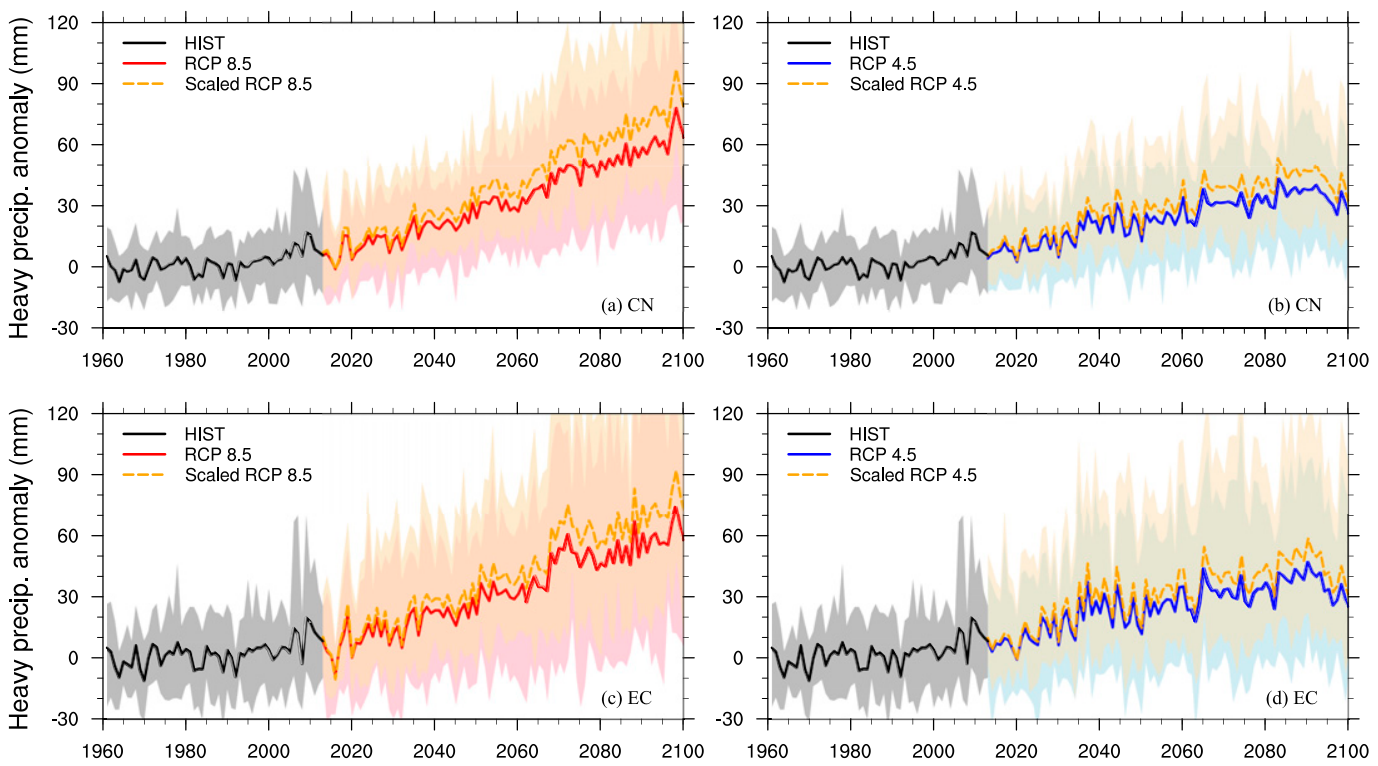

FIG. 7. Changes in heavy precipitation anomalies (relative to 1961-90) for 1961-2100 in (a),(b) China and (c),(d) eastern China. The future climate is projected on the basis of the multimodel ensemble means for the (right) RCP4.5 and (left) RCP8.5 scenarios. The dashed lines indicate the best estimates of observation-constrained future heavy precipitation projections. The shaded areas show the $5 \%-95 \%$ ranges of model simulations.

of heavy precipitation in the future. For moderate precipitation, there is no significant trend in the future projections from the CMIP5 models, and light precipitation displays an obvious decreasing trend in the future in China and EC (see Figs. S7 and S8 in the online supplemental material).

We also roughly estimate the relation between the changes of heavy precipitation and the global mean near-surface temperature (GMST) increase. Based on a recent study about GMST changes by $\mathrm{Hu}$ et al. (2017), the GMST will reach $1.5^{\circ} \mathrm{C}$ warming relative to preindustrial around 2025 (center year for a 31-yr average) and reach $4.5^{\circ} \mathrm{C}$ warming relative to preindustrial by 2100 under the RCP8.5 scenario. Correspondingly, based on the RCP8.5 results the summer heavy precipitation averaged in China will increase by approximately $52 \mathrm{~mm}$ from $2025\left(1.5^{\circ} \mathrm{C}\right.$ warming $)$ to $2100\left(4.5^{\circ} \mathrm{C}\right.$ warming), which is approximately $4.1 \% \mathrm{~K}^{-1}$. After applying the observationally constrained method, the increase rate is approximately $5.1 \% \mathrm{~K}^{-1}$. If we use the China annual and summer mean temperature to estimate the precipitation changes with temperature, the rates become lower (Table $\mathrm{S} 2$ in the online supplemental material) because of more rapid warming in China than global average. The scaled change is also larger than the estimates based on direct projection from the models. However, since we only use a fixed threshold of $25 \mathrm{~mm} \mathrm{day}^{-1}$ to define heavy precipitation, the relation between heavy precipitation changes in China and temperature increase still needs further study. All of these results indicate the importance of model bias adjustment in future projections.

\section{Conclusions and discussion}

Using an optimal fingerprinting method, we compared the changes in summer precipitation of different intensities based on observations and CMIP5 simulations for China, EC and WC. We found a substantial increase in summer heavy precipitation in most parts of China. The CMIP5 models generally reproduce the observed increase in heavy precipitation but underestimate the changes in EC. Detection analyses show that the anthropogenic signal can be identified for heavy precipitation in China and EC, whereas the natural signal cannot be detected. For moderate and light precipitation, external forcings cannot be detected individually. When the three categories of precipitation are combined and their changes are considered simultaneously, the human influence can be detected. The anthropogenic influence contributes most of the observed changes in summer precipitation in China.

In addition, CMIP5 models may underestimate the observed increase in heavy precipitation in China and EC. Observationally constrained future projections of summer precipitation are determined in China and EC by multiplying the multimodel ensemble mean by the scaling factors, obtained from the detection and 
attribution analysis. Under different warming scenarios (RCP4.5 and RCP8.5), the heavy precipitation both shows a significant increase in the future, and with somewhat stronger magnitude after being scaled. The results suggest a possible future that would have increased heavy precipitation and a higher risk of extreme precipitation than that based on raw multimodel simulations. This finding suggests that diverse adaptive policies will be needed in China in the future.

Acknowledgments. We thank two reviewers for thoughtful and helpful comments. We acknowledge the Program for Climate Model Diagnosis and Intercomparison and the World Climate Research Programme's Working Group on Coupled Modeling for their roles in making the WCRP CMIP multimodel data sets available. This study was supported by the National Key R\&D Program of China (2018YFC1507702) and the National Natural Science Foundation of China (41775082 and 41675074). Author F. C. Lott was supported by the U.K.-China Research and Innovation Partnership Fund through the Met Office Climate Science for Service Partnership (CSSP) China as part of the Newton Fund, China. Authors N. Christidis and P. A. Stott were supported by the Met Office Hadley Centre Climate Programme funded by BEIS and Defra.

\section{REFERENCES}

Allen, M. R., and W. J. Ingram, 2002: Constraints on future changes in climate and the hydrologic cycle. Nature, 419, 228-232, https://doi.org/10.1038/nature01092.

— , and P. A. Stott, 2003: Estimating signal amplitudes in optimal fingerprinting. Part I: Theory. Climate Dyn., 21, 477-491, https://doi.org/10.1007/s00382-003-0313-9.

,,- J. F. B. Mitchell, R. Schnur, and T. L. Delworth, 2000: Quantifying the uncertainty in forecasts of anthropogenic climate change. Nature, 407, 617-620, https://doi.org/10.1038/ 35036559.

Ban, N., J. Schmidli, and C. Schar, 2015: Heavy precipitation in a changing climate: Does short-term summer precipitation increase faster? Geophys. Res. Lett., 42, 1165-1172, https://doi.org/10.1002/ 2014GL062588.

Bindoff, N. L., and Coauthors, 2013: Detection and attribution of climate change: From global to regional. Climate Change 2013: The Physical Science Basis, T. F. Stocker et al., Eds., Cambridge University Press, 867-952.

Burke, C., and P. Stott, 2017: Impact of anthropogenic climate change on the East Asian summer monsoon. J. Climate, 30, 5205-5220, https://doi.org/10.1175/JCLI-D-16-0892.1.

$\longrightarrow,-$, Y. Sun, and A. Ciavarella, 2016: Attribution of extreme rainfall in southeast China during May 2015. Bull. Amer. Meteor. Soc., 97, S92-S96, https://doi.org/10.1175/BAMSD-16-0144.1.

Chen, H. P., and J. Q. Sun, 2017: Contribution of human influence to increased daily precipitation extremes over China. Geophys. Res. Lett., 44, 2436-2444, https://doi.org/10.1002/ 2016 GL072439.
Christidis, N., and P. A. Stott, 2015: Extreme rainfall in the United Kingdom during winter 2013/14: The role of atmospheric circulation and climate change. Bull. Amer. Meteor. Soc., 97, S46-S50, https://doi.org/10.1175/BAMS-D-15-00094.1.

CMA, 2012: GB/T28592: Grade of precipitation (in Chinese). China Meteorological Association, 2 pp.

— 2016: China Climate Bulletin 2016 (in Chinese with English abstract). China Meteorological Administration, 50 pp., https:// www.cma.gov.cn/root7/auto13139/201701/t20170117_460484.html.

_ 2017: China Climate Bulletin 2017 (in Chinese with English abstract). China Meteorological Administration, 54 pp., https://www.cma.gov.cn/root7/auto13139/201801/t20180117_ 460484.html.

Donat, M. G., L. V. Alexander, N. Herold, and A. J. Dittus, 2016: Temperature and precipitation extremes in century-long gridded observations, re-analyses, and atmospheric model simulations. J. Geophys. Res. Atmos., 121, 11174-11189, https://doi.org/ 10.1002/2016JD025480.

Fu, C., and L. Dan, 2014: Trends in the different grades of precipitation over South China during 1960-2010 and the possible link with anthropogenic aerosols. Adv. Atmos. Sci., 31, 480491, https://doi.org/10.1007/s00376-013-2102-7.

Hardwick Jones, R., S. Westra, and A. Sharma, 2010: Observed relationships between extreme sub-daily precipitation, surface temperature, and relative humidity. Geophys. Res. Lett., 37, L22805, https://doi.org/10.1029/2010GL045081.

Hu, T., Y. Sun, and X. Zhang, 2017: Temperature and precipitation projection at $1.5^{\circ}$ and $2{ }^{\circ} \mathrm{C}$ increase in global mean temperature (in Chinese). Chin. Sci. Bull., 62, 3098-3111, https://doi.org/ 10.1360/N972016-01234.

Huntingford, C., 2013: Refining global warming projections. Nat. Climate Change, 3, 704-705, https://doi.org/10.1038/nclimate1964.

Jones, G. S., P. A. Stott, and F. B. Mitchell, 2016: Uncertainties in the attribution of greenhouse gas warming and implications for climate prediction. J. Geophys. Res. Atmos., 121, 69696992, https://doi.org/10.1002/2015JD024337.

Li, C., and Coauthors, 2018: Attribution of extreme precipitation in the lower reaches of the Yangtze River during May 2016. Environ. Res. Lett., 13, 014015, https://doi.org/10.1088/17489326/aa9691.

Li, H., H. Chen, and H. Wang, 2017: Effects of anthropogenic activity emerging as intensified extreme precipitation over China. J. Geophys. Res. Atmos., 122, 6899-6914, https:// doi.org/10.1002/2016JD026251.

Ma, S. M., T. J. Zhou, A. Dai, and Z. Han, 2015: Observed changes in the distributions of daily precipitation frequency and amount over China from 1960 to 2013. J. Climate, 28, 69606978, https://doi.org/10.1175/JCLI-D-15-0011.1.

_ - and Coauthors, 2017: Detectable anthropogenic shift toward heavy precipitation over eastern China. J. Climate, 30, 13811396, https://doi.org/10.1175/JCLI-D-16-0311.1.

Min, S.-K., X. Zhang, and F. Zwiers, 2008: Human-induced Arctic moistening. Science, 320, 518-520, https://doi.org/10.1126/ science.1153468.

,,,--- P. Friederichs, and A. Hense, 2009: Signal detectability in extreme precipitation changes assessed from twentieth century climate simulations. Climate Dyn., 32, 95111, https://doi.org/10.1007/s00382-008-0376-8.

,,,--- and G. C. Hegerl, 2011: Human contribution to more-intense precipitation extremes. Nature, 470, 378-381, https://doi.org/10.1038/nature09763.

Pall, P., T. Aina, D. A. Stone, P. A. Stott, T. Nozawa, A. G. J. Hilberts, D. Lohmann, and M. R. Allen, 2011: Anthropogenic 
greenhouse gas contribution to flood risk in England and Wales in autumn 2000. Nature, 470, 382-385, https://doi.org/ 10.1038/nature09762.

Qian, W., J. Fu, and Z. Yan, 2007: Decrease of light rain events in summer associated with a warming environment in China during 1961-2005. Geophys. Res. Lett., 34, L11705, https:// doi.org/10.1029/2007GL029631.

Ribes, A., and L. Terray, 2013: Application of regularised optimal fingerprint to attribution. Part II: Application to global nearsurface temperature. Climate Dyn., 41, 2837-2853, https:// doi.org/10.1007/s00382-013-1736-6.

Semenov, V. A., and L. Bengtsson, 2002: Secular trends in daily precipitation characteristics: Greenhouse gas simulation with a coupled AOGCM. Climate Dyn., 17, 123-140, https://doi.org/ 10.1007/s00382-001-0218-4.

Stott, P. A., and G. S. Jones, 2012: Observed 21st century temperatures further constrain likely rates of future warming. Atmos. Sci. Lett., 13, 151-156, https://doi.org/10.1002/asl.383.

, J. F. Mitchell, M. R. Allen, T. L. Delworth, J. M. Gregory, G. A. Meehl, and B. D. Santer, 2006: Observational constraints on past attributable warming and predictions of future global warming. J. Climate, 19, 3055-3069, https://doi.org/ 10.1175/JCLI3802.1.

Sun, Y., S. Dong, T. Hu, X. Zhang, and P. A. Stott, 2019: Anthropogenic influence on the heaviest June precipitation in southeastern China since 1961. Bull. Amer. Meteor. Soc., 100, S79-S83, https://doi.org/10.1175/BAMS-D-18-0114.1.

Taylor, K. E., R. J. Stouffer, and G. A. Meehl, 2012: An overview of CMIP5 and the experiment design. Bull. Amer. Meteor. Soc., 93, 485-498, https://doi.org/10.1175/BAMS-D-11-00094.1.

Trenberth, K. E., 1998: Atmospheric moisture residence times and cycling: Implications for rainfall rates with climate change. Climatic Change, 39, 667-694, https://doi.org/10.1023/ A:1005319109110.

— - A. Dai, R. M. Rasmussen, and D. B. Parsons, 2003: The changing character of precipitation. Bull. Amer. Meteor. Soc., 84, 1205-1218, https://doi.org/10.1175/BAMS-84-9-1205.

— L. L. Smith, T. Qian, A. Dai, and J. Fasullo, 2007: Estimates of the global water budget and its annual cycle using observational and model data. J. Hydrometeor., 8, 758-769, https:// doi.org/10.1175/JHM600.1.

Wang, X. L., and Y. Feng, 2010: RHtestsV3 User Manual. Environment Canada Science and Technology Branch Atmospheric Science and Technology Directorate Climate Research Division Rep., 27 pp., http://groupware.sinanet.isprambiente.it/scidip/library/ ts-homogeneization_repinfo/r-scritp-user-manual/download/ 1/RHtestsV3_UserManual.pdf.

Westra, S., L. V. Alexander, and F. W. Zwiers, 2013: Global increasing trends in annual maximum daily precipitation. J. Climate, 26, 3904-3918, https://doi.org/10.1175/JCLI-D-12-00502.1.

Wu, P., N. Christidis, and P. Stott, 2013: Anthropogenic impact on Earth's hydrological cycle. Nat. Climate Change, 3, 807-810, https://doi.org/10.1038/nclimate1932.

Xu, W. H., Q. Li, X. L. Wang, S. Yang, L. Cao, and Y. Feng, 2013: Homogenization of Chinese daily surface air temperatures and analysis of trends in the extreme temperature indices. J. Geophys. Res. Atmos., 118, 9708-9720, https://doi.org/ 10.1002/jgrd.50791.

Yang, S., and Q. X. Li, 2014: Improvement in homogeneity analysis method and update of China precipitation data (in Chinese with English abstract). Adv. Climate Change Res., 10, 276-281, https://doi.org/10.3969/j.issn.1673-1719.2014.04.008.

Zhai, P. M., X. Zhang, H. Wan, and X. Pan, 2005: Trends in total precipitation and frequency of daily precipitation extremes over China. J. Climate, 18, 1096-1108, https://doi.org/10.1175/ JCLI-3318.1.

Zhang, J., and T. Zhao, 2019: Historical and future changes of atmospheric precipitable water over China simulated by CMIP5 models. Climate Dyn., 52, 6969-6988, https://doi.org/10.1007/ s00382-018-4559-7.

— — - A. Dai, and W. Zhang, 2019: Detection and attribution of atmospheric precipitable water changes since the 1970 s over China. Sci. Rep., 9, 17609, https://doi.org/10.1038/s41598-01954185-z.

Zhang, X. B., F. W. Zwiers, G. C. Hegerl, F. H. Lambert, N. P. Gillett, S. Solomon, P. A. Stott, and T. Nozawa, 2007: Detection of human influence on twentieth-century precipitation trends. Nature, 448, 461-465, https://doi.org/10.1038/nature06025.

—, H. Wan, F. W. Zwiers, G. C. Hegerl, and S. K. Min, 2013: Attributing intensification of precipitation extremes to human influence. Geophys. Res. Lett., 40, 5252-5257, https://doi.org/ 10.1002/grl.51010.

Zhao, T., C. Li, and Z. Zuo, 2016: Contributions of anthropogenic and external natural forcings to climate changes over China based on CMIP5 model simulations. Sci. China Earth Sci., 59, 503-517, https://doi.org/10.1007/s11430-015-5207-2. 